\title{
A Mass-Temperature Decoupled Discretization Strategy for Large-Scale Molecular-Level Kinetic Model
}

\author{
Zhengyu Chen ${ }^{1}$, Dong Guan ${ }^{1}$, Xiaojie Zhang ${ }^{1}$, Ying Zhang ${ }^{1}$, Suoqi Zhao ${ }^{2}$, Quan Shi $^{3}$, \\ Chunming $\mathrm{Xu}^{4}$, and Linzhou Zhang ${ }^{1}$ \\ ${ }^{1}$ China University of Petroleum Beijing \\ ${ }^{2}$ China University of Petroleum \\ ${ }^{3}$ China University of Petroleum-Beijing \\ ${ }^{4}$ Chinese University of Petroleum
}

February 17, 2021

\begin{abstract}
The molecular conversion of complex mixture involves a large number of species and reactions. The corresponding kinetic model is consist of a series of ordinary differential equations (ODEs) with severe stiffness, leading to an exponentially growing computational time. To reduce the computational time, we proposed a mass-temperature decoupled discretization strategy for a large-scale molecular-level kinetic model. The method separates the mass balance and heat balance calculations in the rigorous adiabatic reactor model and divided the reactor into several isothermal segments. After discretization, the differential equations for heat balance can be replaced by algebraic equations between nodes. We used a molecular-level diesel hydrotreating kinetic model as the case to validate the proposed method. We investigated the effects of temperature estimation methods and node number on the accuracy of the model. A good agreement between the discretization model and rigorous model was observed while the computational time was significantly shortened
\end{abstract}

\section{Hosted file}

Decoupled discretization model-manuscript-20210210.docx available at https://authorea.com/ users/396392/articles/509589-a-mass-temperature-decoupled-discretization-strategy-forlarge-scale-molecular-level-kinetic-model 